\title{
Prospects for Flourishing in Contemporary Health Care
}

\author{
Stephen Pattison ${ }^{1} \cdot$ Andrew Edgar ${ }^{2}$
}

Published online: 8 February 2016

(C) Springer Science+Business Media New York 2016

\begin{abstract}
This special issue of Health Care Analysis originated in an conference, held in Birmingham in 2014, and organised by the group Think about Health. We introduce the issue by briefly reviewing the understandings of the concept of 'flourishing', and introducing the contributory papers, before offering some reflections on the remaining issues that reflection on flourishing poses for health care provision.
\end{abstract}

Keywords Flourishing $\cdot$ Health care $\cdot$ Compassion $\cdot$ Well-being $\cdot$ Resilience

Like 'well-being', flourishing is one of those interesting, historically-significant philosophical concepts that appears to have transferred itself into many areas of life lately, not least into public health care services. The concept of 'flourishing' has played a role in philosophical and ethical debate since the time of Aristotle, at least if his subtle and complex concept of 'eudaimonia' is translated as 'flourishing'. Its very longevity suggests that it is not just a useful concept, but one that needs to be reconsidered for each generation. Our objective here is thus to begin to reconsider 'flourishing' at a time when public health care services in all Western developed countries are under increased pressure to save money, to deliver more service to ageing populations, to be more responsive to user interests and preferences, to adopt advances in medicine, and to show more compassion and care, amongst many other demands.

Stephen Pattison

s.pattison.1@bham.ac.uk

1 Department of Theology and Religion, University of Birmingham, ERI Building, Edgbaston, Birmingham B15 2TT, UK

2 Centre for Applied Ethics, Cardiff University, John Percival Building, Colum Drive, Cardiff CF10 3EU, UK 
If flourishing is understood to mean having a good time, being a wealthy chooser/consumer and enjoying abundant life in all its fullness in a simplistic, pleasure-seeking way, this is not the most propitious time for this concept to reemerge into health care discourse. If, on the other hand, flourishing can be understood more as a quality of positive resistance and resilience that comes into its own when the going gets difficult, then there is no more apposite idea to explore in health care today. Despite the very positive advances in health and well being that Western societies have enjoyed over the last couple of centuries, pressure, illness, suffering and death have not gone away. Flourishing in the face of these factors is a challenge to all, users, workers, and society as a whole.

This special edition of Health Care Analysis emerges from a small symposium held in 2014 organised by the network, Think About Health. Think About Health aims to undertake 'intellectual plumbing', i.e. to provide clear thinking, within health care. The event occurred as the effects of world recession and policies of austerity were really beginning to bite into public health services, and much pessimism was abroad. In this ongoing context, the symposium, and the articles contained herein, sought to ask, What would it mean to flourish in difficult times for the various actors using and working in health care services? As might be expected, flourishing proved to be both an essential and an elusive concept to pin down, by turns vital and apparently veiled. It is in this context that the authors contributing to this special edition tackle and question the nature of flourishing, providing a kaleidoscopic and partial picture of the place and nature of flourishing at various points in the process of life, illness and death.

Valerie Iles, a trainer and management educator in health care, draws on her own personal experience of caring to raise fundamental questions about what it means for patients and their carers to flourish in the context of the end of life. Characterising dying as possibly 'the most grown-up thing' we have to do, Iles considers the ways in which the organisation of life and health services can conspire to make us incapable of grasping the possibilities for life, and so for flourishing, at the moment when this might most matter. A kind of perpetuation of adolescence can de-skill and distort people facing the fundamental facts and experiences of life and death. But if these can be addressed, then a kind of flourishing is possible, even at the limits of human existence. This provides an important experiential horizon against which to evaluate the nature and importance of flourishing.

Flourishing is not just a desirable quality for individuals at the end of life or at the edges of experience. Mary Morton, a psychotherapist, argues in her paper that rupture and repair which inevitably involves pain and suffering, is a necessary part of the process of becoming and remaining a human in society. To flourish, then, is to be able to endure and learn from endless experiences of rupture and repair how to build good enough individual and social relationships and projects. Morton applies this thought to health care systems and to societies, as well as to the individual lives from which this insight emerges in psychological observation of infants and their mothers, suggesting that the occasion and recognition of rupture opens up opportunities for growth and development that need to be welcomed, not feared and avoided, if there is to be a holistic experience of the possibilities of human existence. 
Hugh Middleton argues from the perspective of psychiatry. He identifies links between the idea of flourishing and that of self-actualisation, as developed by Maslow, Rogers and others. Recognising such links opens the possibility, Middleton argues, of there being reliable empirical measures of flourishing. Examining the resultant empirical evidence nevertheless throws up surprising results. Relatively few supposedly healthy people flourish, and yet signs of flourishing are readily identifiable amongst sufferers of posttraumatic stress disorder. Significantly for the question of how a health service can promote flourishing, Middleton argues that health care provision, drawing as it continues to do on the model of the sick role, which renders the patient passive before the ministrations of physicians, is actively inhibiting patient flourishing.

Joao Calinas, a general practitioner, explores the relationship between flourishing and freedom, with particular attention being given to the challenges of chronic illness. Negative conceptions of disease, that conceive of disease as the absence of health, may presuppose that disease serves to reduce a person's freedom, and, as such, their possibility of flourishing. The patient is in effect thereby situated as a failed healthy person. This poses a fundamental problem for how the chronically ill person can live well. Calinas challenges this presupposition by outlining three possible relationships between freedom and flourishing (inspired by a metaphor from Nietzsche). 'Flourishing within freedom' entails that one's capacity to flourishing is indeed restricted by the burden of one's illness. 'Flourishing through freedom' demands a more courageous confrontation of those limitations. 'Flourishing beyond freedom' takes this confrontation further. The patient creatively finds ways to flourish, living well with their condition, and thereby becomes a successful patient rather than a failed healthy person. Akin to Middleton, Calinas thereby challenges certain orthodox approaches to health care provision, as inhibiting rather than facilitating the patient's flourishing.

Finally Edgar and Pattison offer a philosophical reflection on the concept of 'flourishing'. They argue that 'flourishing' is a concept that is uniquely able to engage with the vulnerability of human existence, and as such captures the relationship between flourishing and the experience of the patient and carer. Like other contributors to this issue, they argue that flourishing does not occur in the absence of material or bodily deprivation, but is rather stimulated by the challenges that our human vulnerability to accident, disease and contingency present to us. Ultimately, to flourish, they argue, is to be able to tell a meaningful, but not necessarily happy, story about one's life. The person who is overcome by grief or suffering, and thus whose life loses meaning, cannot go on. They cannot make intelligible choices about the rest of their lives. To flourish presupposes the capacity to go on, meaningfully and realisically.

The papers included here provide useful and partial perspectives on the whole issue of flourishing, but they by no means exhaust understandings of the importance and elusiveness of this concept. Many questions can be asked in light of the issues raised by the contributions above.

At a theoretical level, it can be asked, what is the difference between living, surviving and flourishing? To address this question would require further consideration of the relationship of flourishing to such qualities and activities as 
vulnerability, dependency and nurturing, as well addressing the virtue of courage (a virtue for patients and their carers alike). Philosophically, flourishing has been seen both as a state of being, and as such the goal or telos of human life, and also as an active quality of becoming, and thus striving towards one's goal. In focusing on flourishing in the face of adversity, the papers in this collection emphasise the latter conception of flourishing over the former. In situating flourishing in the context of struggle, illness and hardship, and as such the struggle to flourish, they perhaps neglect the possibility that there are meaningful states of flourishing, or even abounding, that exist when people are enjoying life free from illness and death.

The complexity and subtlety, not merely of the concept but also the experience of flourishing, raises the question as to whether flourishing means the same thing for everyone, or is it a largely contextual phenomenon that is structured, recognised and judged only in particular situations and relational webs. What it is to flourish may differ significantly, both within a culture and between cultures. Even within an individual's life, what counts as flourishing at a particular life stage, or at a particular point within the developing narrative of a life, may vary. Flourishing may not even be something that can be identified as a quality of a whole human life course in all its stages, but rather a quality that emerges only at particular points.

The most fundamental practical question is, whose responsibility is it, then, to promote and identify flourishing in the world? More precisely, it can be asked, is it the job of public health services to promote and ensure flourishing, even to guarantee it, or is it really a wider human task in which the health care services are only involved incidentally? If they do have a role, for whom should they be promoting flourishing (patients, staff, the citizenry in general), and at what level, e.g., at governmental level, at city level, in professional-patient encounters? And what would it mean and look like for those involved at those levels? Given the complexity and diversity of the experience of flourishing, practical problems remain as to how flourishing can be enabled, elicited or encouraged if it is deemed essential and desirable in human life. In over-determined and compressed clinical encounters, the demand to to identify, foster and evaluate flourishing may be an unrealistic demand (and ironically one that may inhibit the flourishing of the carers themselves).

Clearly, the insights and questions presented here provide the merest starting point for exploring a complex quality and concept. However, they point to theoretical and practical debates which go to the heart of health care provision and its purpose. If flourishing is a precious quality of individual and institutional lives, as it seems to be, albeit that it often emerges in difficult times and circumstances, a good deal more attention needs to be accorded it. Without the capacity to flourish in the face of limitations and difficulties, sometimes cruel, often arbitrary, it is difficult to see what life, let alone health services, have to offer. Attending to the nature and experiences of flourishing in the spirit of seeking and promoting phronesis, practical wisdom, may then be a salutary corrective to tendencies towards consumerist rational instrumentality that threaten to overwhelm health care services ideologically at the present time. If we are all going to suffer and die, then we, sufferers, carers and professionals alike, all need better to understand and be able to develop qualities and capacities of flourishing, as much now as in the past. 